\title{
Estructura de bandas en un cristal fotónico unidimensional y bidimensional
}

\section{Band structure in a photonic crystal one dimensional and two dimensional}

\author{
José Luis León Aguirre \\ Universidad Nacional del Callao
}

DOI: https://doi.org/10.33017/RevECIPeru2015.0003/

Resumen

Una nueva idea se ha estado desarrollando en las últimas décadas, cuyo objetivo es lograr control de las propiedades ópticas de materiales con el fin de resolver ciertos problemas sujetos a nuevas aplicaciones, como por ejemplo: obtener materiales que respondan a las ondas de luz sobre un rango de frecuencias deseados para obtener su total reflexión. Este trabajo se enfoca en el desarrollo matemático del problema de las estructuras de bandas de un cristal fotónico, utilizando el método de expansión de ondas planas a los casos particulares de una y dos dimensiones.

\begin{abstract}
A new concept has been developing in recent decades, whose aim is to achieve control of the optical properties of materials in order to solve certain problems subject to new applications, such as for example: To obtain materials to meet the waves of light on a range of desired frequencies to get your total reflection. This work focuses on the mathematical development of the problem of the structures of bands of a photonic crystal, using the method of expansion of flat waves to individual cases of one and two dimensions.
\end{abstract}

\section{Introducción}

Un cristal fotónico es análogo a los cristales electrónicos, en el cual los átomos o moléculas son remplazados por un medio dieléctrico y un potencial periódico. Estos materiales tienen efectos sorprendentes, si se tiene un diseño y construcción de tal forma que el dieléctrico del material sea diferente de tal forma que la refracción y reflexión de la luz en las múltiples interfaces puedan producir para los fotones muchos de los fenómenos conocidos como brechas completas o bandas prohibidas.

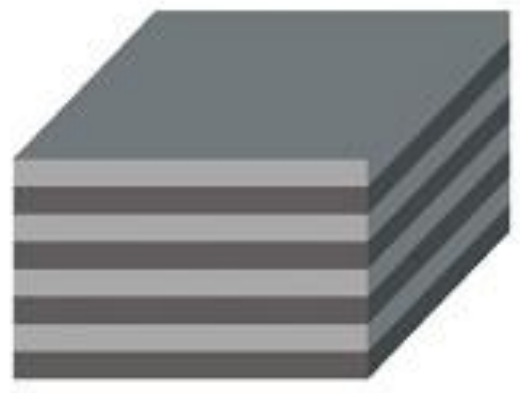

1D 
Se tiene que:

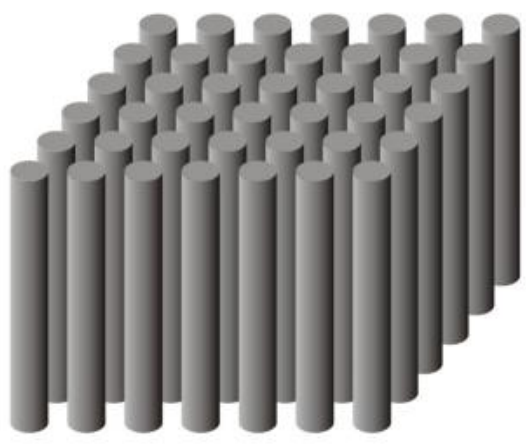

2D

\section{Red recíproca}

En el análisis de los cristales fotónicos, nos concentramos en el espacio directo $\mathrm{R}$ y el concepto de la red recíproca a la red en el espacio de frecuencias, es decir definida por los vectores $G$, los cuales llamamos vectores de la red recíproca.

Los vectores de la red reciproca $\mathrm{R}$ pueden escribirse en términos de los vectores primitivos de la red reciproca $\widehat{\boldsymbol{a}_{\boldsymbol{t}}}$ con vectores unitarios y parámetro de red a, de manera que:

$$
R=\sum_{i=1} l a_{i}=l a \widehat{a_{1}}+n a \widehat{a_{2}}+m a \widehat{a_{3}}
$$

De forma análoga, los vectores de la red reciproca $G$ pueden escribirse en términos de los vectores primitivos de la red reciproca $\widehat{\boldsymbol{b}}_{\boldsymbol{\imath}}$ con vectores unitarios y parámetro de red $\mathrm{b}$, de manera que:

$$
b=\left\{\sum_{i=1}^{n} B_{i} \widehat{b}_{l}\right\}
$$

Donde $\left\{\boldsymbol{b}_{1} \ldots \ldots \boldsymbol{b}_{\boldsymbol{n}}\right\}$ es una base del espacio de frecuencias rectangular $\boldsymbol{K}^{n} \operatorname{con} \boldsymbol{B}_{\boldsymbol{i}}$ entero

Además:

$$
G=\sum_{i=1}^{n} L \widehat{b_{l}}=L b \widehat{b_{1}}+N b \widehat{b_{2}}+M b \widehat{b_{3}}
$$

Conociendo la forma de R y $G$, y debido al requerimiento:

$$
G \cdot R=2 \pi n
$$

$G \cdot R=\left(l a_{1}+n a_{2}+m a_{3}\right) \cdot\left(L b_{1}+N b_{2}+M b_{3}\right)=2 \pi n^{\prime}$

Dada la ortonormalidad de los vectores $\widehat{\boldsymbol{a}}_{\boldsymbol{l}}$ y $\widehat{\boldsymbol{b}}_{\boldsymbol{l}}$, de forma intuitiva podríamos construir vectores $\widehat{\boldsymbol{b}}_{\boldsymbol{\imath}}$ de manera que se cumpla la siguiente relación:

$$
a_{i} \cdot b_{j}=2 \pi \delta_{i j}
$$

Ahora, para encontrar los vectores $\widehat{\boldsymbol{b}_{\boldsymbol{l}}}$, dado un en función de los vectores $\widehat{\boldsymbol{a}_{\boldsymbol{l}}}$ :

$$
b_{1}=\frac{2 \pi\left(a_{2} \times a_{3}\right)}{a_{1 .}\left(a_{2} \times a_{3}\right)}
$$

$$
b_{2}=\frac{2 \pi\left(a_{3} \times a_{1}\right)}{a_{1 .}\left(a_{2} \times a_{3}\right)}
$$

$$
b_{3}=\frac{2 \pi\left(a_{1} \times a_{2}\right)}{a_{1 .}\left(a_{2} \times a_{3}\right)}
$$

\section{Funciones periódicas en el espacio de frecuencias}

Una función $f(r)$ es periódica si cumple la condición de periodicidad dada por:

$$
f(r)=f(r+R)
$$

$R$ es el periodo de la función.

Además $\mathrm{R}$ se puede escribir en términos de los vectores primitivos a que a su vez pueden ser escritos en términos de los vectores unitarios ai y el parámetro de red a, de tal forma que para una red cúbica en las tres dimensiones se tiene:

$$
R=\sum_{i=1} l a_{i}=l a \widehat{a_{1}}+l a \widehat{a_{2}}+l a \widehat{a_{3}}
$$

Se expresar la función $f(r)$ como una sumatoria ponderada sobre todos los vectores de red recíproca, de manera que:

$$
f(r)=\sum_{G} f(G) e^{i G . r}
$$

Donde $\mathrm{G}$ es la red recíproca. 
condiciones necesarias para el problema de

\section{Condición de difracción}

En dicho sistema se identifica claramente una disposición de red puntual en la que los nodos contienen un medio con una constante dieléctrica diferente a la del resto del espacio; dado que e(r) es una función periódica, puede ser escrita como:

$$
\varepsilon(r)=\sum_{G} \varepsilon(G) e^{i G . r}
$$

Intuitivamente se puede suponer que la amplitud de la onda dispersada por un elemento de volumen $\mathrm{dV}$ con vector de onda $\mathrm{k}$ ' depende directamente de la constante dieléctrica local, de manera que la amplitud total de la onda dispersada $A$ esta dada por la integral y la diferencia de k y k', se expresa matemáticamente de la siguiente forma:

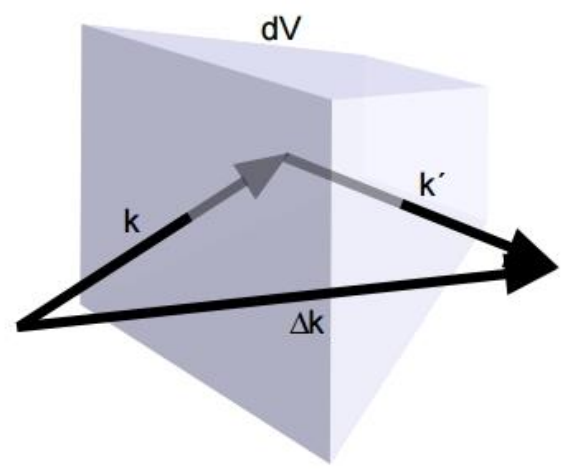

Dispersión de una onda plana (gráfico)

$$
A=\sum_{G} e^{-i(G-\Delta K)} \varepsilon(G) d V
$$

Si se considera dispersión elástica para los fotónes, de tal forma que su energía se conserva, entonces se obtiene la siguiente ecuación:

$$
K=\mp G / 2
$$

\section{Fundamentos y propiedades de los cristales fotónicos}

Para determinar la banda prohibida analizaremos las ecuaciones de Maxwell y además las autovalores.

- Se trata de un material transparente real y positivo.

- No hay carga ni corriente libre.

- Se considera un material macroscópico e isotrópico.

$$
\begin{gathered}
\nabla \cdot\{\varepsilon(r) E(r, t)\}=0 \\
\nabla \times\{E(r, t)\}=-\mu_{0} \frac{\partial H(r, t)}{\partial t} \\
\nabla \cdot\{H(r, t)\}=0 \\
\nabla \times\{H(r, t)\}=\varepsilon_{0} \frac{\partial E(r, t)}{\partial t}
\end{gathered}
$$

Además considerando que varía sinusoidalmente con el tiempo

$$
\begin{aligned}
& E(r, t)=E(r) e^{-i \omega t} \\
& H(r, t)=H(r) e^{-i \omega t}
\end{aligned}
$$

Reorganizando, obtenemos:

$$
\nabla \times\left\{\frac{1}{\varepsilon(r)} \nabla \times H(r)\right\}=\left(\frac{\omega^{2}}{c^{2}}\right) H(r) \ldots(1)
$$

Nos enfocamos en encontrar la frecuencia de los modos, estamos en un problema de autovalores. Los modos electromagnéticos pueden ser escritos como estados de Bloch:

$$
H_{k}(r)=e^{i k . r} \mu_{k}(r) . .(2)
$$

De la ecuación (2) en (1) se obtiene: 


$$
\begin{array}{r}
\left\{(i k+\nabla) \times \frac{1}{\varepsilon(r)}(i k+\nabla) \times u_{k}(r)\right\} \\
=\left(\frac{\omega(k)^{2}}{c^{2}}\right) u_{k}(r) . .
\end{array}
$$

\section{El electromagnetismo como un problema de autovalores}

De las ecuación (3) es claramente una ecuación de autovalores y autovectores que tiene la forma:

$$
\widehat{\sigma} v=\lambda v
$$

Además como se aprecia en la ecuación 3 vemos un conjunto de operaciones que actúan en $\boldsymbol{H}(\boldsymbol{r})$ y los autovalores $\left(\frac{\omega}{c}\right)^{2}$, puede ser escrita como:

$$
\widehat{\Theta} H(r)=\left(\frac{\omega}{c}\right)^{2} H(r)
$$

Los autovectores de $\boldsymbol{H}(\boldsymbol{r})$ son los patrones espaciales de los modos armónicos donde los autovalores $\left(\frac{\omega}{c}\right)^{2}$ son proporcionales a las frecuencias de dichos modos.

Los estados del hamiltoniano poseen algunas propiedades importantes: sus autovalores son reales, son autoestados ortogonales y pueden ser calificados por su propiedad de simetría.

La formulación electromagnética, debemos definir el producto interno de dos funciones de campo:

$$
(F, G)=\int_{v} F^{*}(r) \cdot G(r) d^{3} r
$$

Además debe cumplir lo siguiente:

$$
F(r)=\frac{F^{\prime}(r)}{\sqrt{\left(F^{\prime}(r), F^{\prime}(r)\right)}}
$$

Con el fin de normalizar los modos.

Ahora, verificar la hermiticidad de $\widehat{\boldsymbol{\Theta}}$ :

$$
(F, \widehat{\Theta} G)=\int_{v} F^{*} \cdot \nabla \times\left(\frac{1}{\epsilon} \nabla \times G\right) d^{3} r
$$

Luego haciendo un cambio de variable

$$
A=F^{*}
$$

$$
B=\frac{1}{\epsilon} \nabla \times G
$$

Se obtiene la siguiente ecuación:

$$
\begin{aligned}
\int_{v} A \cdot(\nabla \times B) d^{3} r & \\
& =\int_{v}(\nabla \times \mathrm{A}) \cdot \mathrm{B} d^{3} r-\int_{s}(A \times B) \cdot d^{2} r
\end{aligned}
$$

Además, las fronteras de integración pueden despreciarse debido a que los campos decaen a cero a grandes distancias (modos evanescentes), entonces los términos de la superficie desaparecen de modo que:

$$
\int_{S} A \times B \cdot d^{2} r=0
$$

Se reduce a:

$$
\begin{aligned}
& \int_{v} A \cdot(\nabla \times B) d^{3} r=\int_{v}(\nabla \times A) \cdot B d^{3} r \\
& (F, \hat{\Theta} G)=\int_{v}(\nabla \times F)^{*} \cdot\left(\frac{1}{\epsilon} \nabla \times G\right) d^{3} r
\end{aligned}
$$

Se repite el mismo procedimiento con los siguientes cambios de variable:

$$
A=\frac{1}{\epsilon}(\nabla \times F)^{*}
$$

$$
B=G
$$

Para obtener:

$$
(F, \widehat{\Theta} G)=\int_{v}\left(\nabla \times\left(\frac{1}{\epsilon} \nabla \times F\right)\right)^{*} \cdot G d^{3} r
$$


De modo que el operador $\widehat{\boldsymbol{\Theta}}$ es un operador Hermítico ya que:

$$
\widehat{\Theta}^{*}=\hat{\Theta}
$$

\section{Método de expansión de ondas planas}

Se expresa la función dieléctrica y función periódica de un cristal fotónico unidimensional y bidimensional y expresar aquellas funciones como una expansión en series de Fourier.

Unidimensional

$$
\begin{gathered}
\varepsilon(z)=\sum_{G} \varepsilon(G) e^{i G . z} \\
u(z)=\sum_{G} u(G) e^{i G . z} \widehat{a_{y}}
\end{gathered}
$$

\section{Bidimensional}

$$
\begin{aligned}
& \varepsilon(x, y)=\sum_{G_{x}^{\prime}} \sum_{G_{y}^{\prime}} \varepsilon\left(G_{x}^{\prime}, G_{y}^{\prime}\right) e^{i G_{x}^{\prime} \cdot x} e^{i G^{\prime} y \cdot y} \\
& u(x, y)=\sum_{G_{x}} \sum_{G_{y}} u\left(G_{x}, G_{y}\right) e^{i G_{x} \cdot x} e^{i G_{y} \cdot y} \widehat{a_{z}}
\end{aligned}
$$

Reemplazando las funciones expresadas en serie de Fourier en la ecuación (3), se obtiene:

\section{Unidimensional}

$$
\left(k^{\prime}+n\right)^{2} u(n)=\sum_{n^{\prime}} \varepsilon\left(n-n^{\prime}\right) u(n)
$$

\section{Bidimensional}

$$
\begin{aligned}
\left\{\left(k^{\prime}{ }_{x}+n\right)^{2}+\left(k^{\prime}{ }_{y}+m\right)^{2}\right\} u(n, m) & \\
& =\left(\omega^{\prime 2}\right) \sum_{n^{\prime}} \sum_{m^{\prime}} \varepsilon\left(n-n^{\prime}\right) u(n, m)
\end{aligned}
$$

Se evalúa la magnitud k' para ambas ecuaciones para simular posibles direcciones de la banda prohibida en un cristal fotónico unidimensional y bidimensional.

\section{Diagrama de bandas del cristal fotónico unidimensional y bidimensional}

\section{Unidimensional}

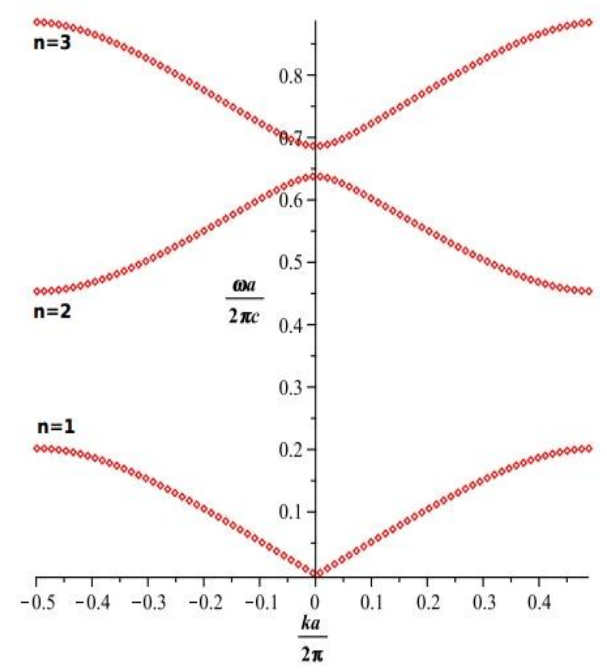

\section{Bidimensional}

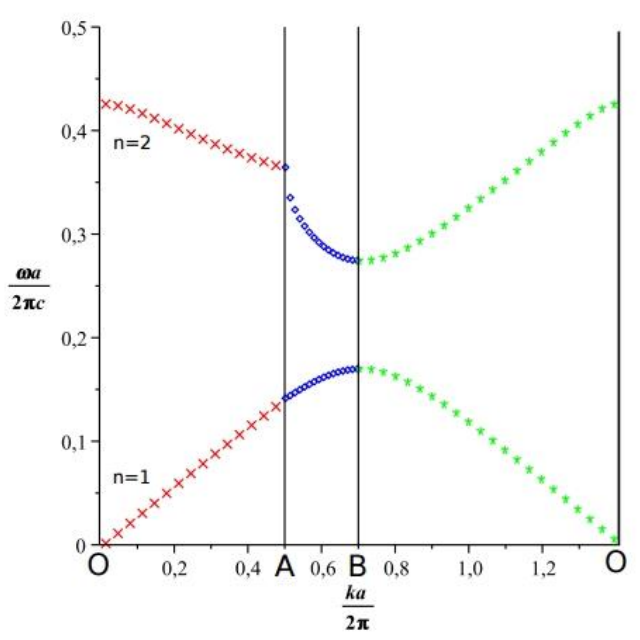




\section{Resultado y discusión}

Se utiliza este tipo de método debido a que los resultados convergen y por tanto la simulación es más eficiente comparado al método de diferencia finitas en el dominio de frecuencias que necesitan de un gran cantidad de iteración. Básicamente se calculan las frecuencias del cristal fotónico para cada valor de dirección de propagación dada por el vector $\mathrm{k}$, además los valores normalizados de los parámetros se deben ajustar para diferencia la banda prohibida la cual es el objetivo del trabajo. Cabe señalar que en los resultados de la simulación sea cual sea la dirección de una onda transversomagnética, si tiene una frecuencia perteneciente a la banda prohibida, no podrá transmitirse a través del cristal. Además la estructura del cristal fotónico influye de manera drástica los resultados de las estructura de las bandas, es decir la banda prohibida se hace más pequeña en distancia de separación, debido a las múltiples direcciones de la onda electromagnética que inciden en el cristal fotónico.

\section{Conclusión}

Se estudiaron conceptos y métodos para evidenciar las propiedades importantes que tienen los cristales fotónicos con el fin de encontrar la estructura de bandas tanto para modelo unidemensional y bidimensional. Se implementaron métodos la expansión de ondas planas para diferenciar el gap en ambos diagramas de bandas, situación especial en cristales fotónicos unidemensional y dimensional obteniendo diferencias notables en la banda prohibida. En este trabajo se estudia el comportamiento transversoeléctricos en el cristal fotónico, pero la idea fundamental es de extender el estudio para cristales fotónicos tridimensionales para ondas electromagnéticas de diferentes direcciones que inciden al material, para obtener bandas prohibidas omnidireccionales. De igual manera se puede estudiar caso particulares con el fin de evidenciar propiedades más específicas tanto en los sistemas unidemensionales y bidimensionales variar incluso las constantes dieléctricas para obtener cristales con filtros de frecuencia especifica además la inclusión de defectos locales en el cristal con el fin de obtener cavidades resonantes y los defectos que abarquen todo el cristal para obtener sistemas de guiado de ondas.

\section{Referencias}

[1] J. N. Winn, J. D Joannopoulos, S. G. Johnson and R. D. Meade, Photonic Crystals: Molding the Flow of Light, 2nd ed. (2008) 44-65.

[2] K. Yasumoto, Electromagnetic Theory and Applications for Photonic Crystals (2006) 142 164.

[3] J.D. Jackson, Classical Electrodynamics, 3rd ed. (1989) 237-258.

[4] J. W. Goodman, Introduction to Fourier Optics, 3rd ed. (2005) 3-84.

[5] Revista Electrónica de Ciencia, Tecnología, Sociedad y Cultura. ISSN 2174-6850.

E-mail: leon_aguirre_25@hotmail.com 